\title{
COME SCRIVE PLATONE \\ ESEMPI DI UNA SCRITTURA A CARATTERE \\ "PROTRETTICO"
}

Maurizio Migliori ${ }^{1}$

\section{RESUMO}

Vários estudiosos dedicados ao pensamento de Platão defendem a tese segundo a qual o texto do ateniense é incongruente, contraditório e até mesmo "irremediavelmente estranho". Neste artigo, tentamos demonstrar em que medida a crítica ao texto de Platão deve recair não sobre o seu gênio literário ou seu talento filosófico extraordinários - hipótese obviamente absurda -, mas, isso sim, sobre a qualidade do intérprete e do método usado para estudar o pensamento do filósofo. Aqui, entre outras coisas, analisamos, com base em trechos emblemáticos dos próprios diálogos, o conceito platônico de "escrito-jogo", verdadeiramente fundamental para a compreensão das aparentes desarmonias do texto platônico.

PALAVRAS-CHAVE: Erro voluntário. Escrito. Seriedade. Dialética. Educação.

\section{ABSTRACT}

Many scholars dedicated to the Plato's philosophy sustain that atenian's text is incongruous, contradictory and even "irremediable

1 Professor de Storia della Filosofia Antica, Università degli Studi di MacerataUNIMC. 
strange". In this article, we try to demonstrate how the censure to Plato's text must fall again not on his extraordinary literary genious or his philosophical talent - hypothesis clearly absurd -, but, as a matter of fact, on the quality of the interpreter and the method used to understand Plato's philosophical thought. Here, among other things, we analyse, on the basis of emblematic portions extracted of the dialogues, the platonical concept of "writtengame", truly essencial to the comprehension of the apparent disharmony of our philosopher text.

KEYWORDS: Voluntary mistake. Writing. Seriousness. Dialectics. Education.

\section{Alcune premesse di metodo}

Tra le molte cose sconcertanti che caratterizzano gli studi platonici una emerge con particolare spicco: la presenza in vari studiosi dell' "inconscia" convinzione che Platone sia "irrimediabilmente strano" e capace di gesti a volte inesplicabili. In effetti, lo studioso dei dialoghi ha continuamente l'impressione di trovarsi di fronte a svolte e salti (per non dire contraddizioni) che non sembrano giustificati, per non parlare del vero e proprio shock che si prova la prima volta di fronte ai dialoghi "aporetici" o dello smarrimento che si ha sempre di fronte alle testimonianze aristoteliche, come quelle densissime degli ultimi due libri della Metafisica.

Questa "precomprensione" determina spesso un effetto scientificamente poco ammissibile: di fronte ai passi più evidentemente contraddittori si pratica la rimozione, quando non si giunge ad affermazioni sconcertanti del tipo: "Qui Platone ha evidentemente dimenticato quello che ha detto... nella pagina precedente".

L'ipotesi che qui presentiamo è, al limite, opposta: Platone ha un controllo "eccezionale" del suo testo per cui occorre soffermarsi con particolare attenzione proprio là dove emerge l'incongruenza, 
il salto, l'assurdo. La difficoltà che la critica incontra non è connessa primariamente ai temi proposti o ad eventuali limiti espositivi dell' Autore che, come tutti riconoscono, è insieme grandissimo filosofo e grandissimo genio letterario. Il dato distintivo dei dialoghi è che l'Autore, per ragioni eminentemente educative, ha "inventato" e sviluppato una particolare tecnica di scrittura: questa è la caratteristica che distingue Platone da tutti gli altri scrittori di filosofia. Non si tratta affatto di esoterismo, nel senso tradizionale del termine ${ }^{2}$, ma di una particolare concezione teorica: Platone è convinto che la filosofia non si apprende, ma si fa, per cui il maestro deve essere colui che aiuta il soggetto a compiere il suo percorso euristico che è insieme vitale e intellettuale ${ }^{3}$. Pertanto lo scrittore, se vuole essere filosofo, deve "spingere" il lettore a "fare filosofia", non dando "soluzioni" ma indicando i problemi e le vie che portano alla soluzione degli stessi:

2 Non posso approfondire tale questione, connessa alla Scuola di Tubinga-Milano e al "Nuovo paradigma"; rimando a tre articoli in cui ho già svolto questo tipo di riflessioni: M. Migliori, Il recupero della trascendenza platonica e il nuovo paradigma, "Rivista di filosofia neo-scolastica», 79 (1987), pp. 351-381; La scuola di Tubinga-Milano, "Il Cannocchiale», 1992, pp. 121-142; De la critique de Schleiermacher aux commentaires récents. Evolution et articulation du nouveau paradigme de Tübingen-Milan, «Études Philosophiques», 1998, pp. 91-114.

3 Lettera settima, 340 B 7- D 6: «A tali individui bisogna mostrare che cosa sia nel suo insieme tale lavoro, quale caratteristica abbia, quante cose implichi e quanta fatica comporti. Infatti, chi ascolta, se è veramente filosofo, adatto e degno di essa, in quanto dotato di natura divina, pensa che quella che ha sentito sia una via meravigliosa e che si debba intraprendere subito e che non si possa vivere agendo diversamente. Quindi, unendo i suoi sforzi a quelli di colui che lo conduce nella via, non desiste prima di aver raggiunto completamente il fine, o prima di aver acquisito la forza per potere da solo procedere senza uno che lo guidi. In questo modo e secondo questi pensieri vive un uomo siffatto, dedicandosi alle sue faccende, quali che siano, in tutto seguendo sempre la filosofia e quel modo di vivere quotidiano che più di ogni altro lo può rendere capace di apprendere, di ricordare, di ragionare con la piena padronanza di se stesso; passa la vita odiando invece il modo di vivere contrario a questo»; Lettera settima, 341 C 5- D 2: «questa conoscenza non è comunicabile come le altre, ma da molte discussioni su questi problemi e da una comunanza di vita, all'istante, come luce che si sprigiona da una scintilla balzata via, essa nasce nell'anima e da se stessa si alimenta». 
Ma non credo che comunicare la trattazione di questi argomenti sia bene per gli uomini, se non per quei pochi che sono capaci da soli di trovare soluzioni sulla base di poche indicazioni ${ }^{4}$.

Fornire questo aiuto rispettoso dei livelli di maturazione dell' allievo, il dire in modo semplice per anime semplici e complesso per anime complesse, ha evidentemente ben differenti livelli di possibilità nella comunicazione orale e in quella scritta. Nella prima, infatti, l'interlocutore è certo e le "anime" sono a contatto, cosa che è programmaticamente esclusa nell' altra. Per questo un grande scrittore filosofico come Platone imposta una forma di comunicazione, di insegnamento, che definisce sia nella Lettera Settima ${ }^{5}$ sia nel $\mathrm{Fedro}^{6}$, con i termini di attività non seria e di gioco, giungendo ad affermare che è filosofo

colui che ritiene che in un discorso scritto su qualunque argomento vi è necessariamente molta parte di gioco e che non sia mai stata scritta un'opera in versi o in prosa degna di molta serietà7.

Certo si tratta di giochi non futili anzi molto belli ${ }^{8}$, scritti non solo come promemoria ma anche per chi segue la stessa traccia ${ }^{9}$, un impegno tale da potervi dedicare tutta la vita ${ }^{10}$. E tuttavia di giochi si tratta: Platone, proprio perché è convinto socraticamente che la filosofia è lavoro comune e scoperta, dà luogo ad un insegnamento che sempre, ma soprattutto nella forma scritta, avvicina al vero ma senza rivelarlo, comunica informazioni vere

4 Lettera settima, 341 E 1-3.

5 Cfr. anche Lettera Settima, 344 C 3-8.

6 Fedro, 276 C 2-3; 278 D 3-6.

7 Fedro, 277 E 5-8.

8 Fedro, 277 E 1-2.

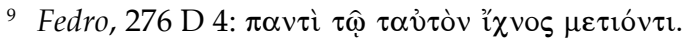

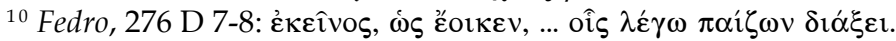

Educ. e Filos., Uberlândia, v. 20, n. 40, p. 41-80, jul./dez. 2006. 
che non sono tout court la verità, ma che richiedono la partecipazione, l'elaborazione e uno sviluppo da parte del lettore. Per così dire, l'Autore imposta un gioco che l'altro deve svolgere. Ciò richiede sempre, e quindi giustifica, una grande "reticenza educativa" del maestro che non vuole impedire al lettore di scoprire la verità comunicandogliela direttamente. In questo senso si può dire che la filosofia platonica scritta è, ad un tempo, non scritta nel senso che, pur in presenza di un pensiero "compiuto", non abbiamo mai una vera e propria "esposizione" neanche di quelle teorie che gli sono universalmente attribuite, come la teoria delle Idee.

In questa chiave di "gioco serio", il corpus platonico appare come un vero e proprio "protrettico" che propone filosofia per costringere il lettore filosofo a "trovare soluzioni sulla base di poche indicazioni", il che implica la proposta di difficoltà crescenti che via via nello svolgimento delle opere selezionano i "veri filosofi".

Se questa è la situazione, non è possibile uscire dalla situazione di "blocco" in cui si trovano gli studi platonici se non si comincia a lavorare in modo fermo e costante sul terreno della tecnica di scrittura del filosofo ateniese. Il problema diviene allora quello di capire, caso per caso, quali sono gli strumenti con cui Platone, muovendosi con assoluta libertà, "gioca" con il lettore ${ }^{11}$. Naturalmente l'interprete deve muoversi con grande prudenza e consapevolezza, per evitare di cadere in continui arbitri: il gioco di Platone non deve essere scoperto dalla "intelligente inventiva" del lettore, ma deve essere esplicito e concretizzarsi in "segnali" testuali.

Cercherò di evidenziare questo dato negli esempi che ora proporrò. Non mi interessa, in questa sede, sostenere una qualche specifica soluzione ma solo mostrare come, prestando la dovuta attenzione alla tecnica di scrittura e scoprendo i "segnali" che

${ }^{11}$ Ho già affrontato questo tipo di riflessione, ma con un taglio ed esempi del tutto diversi, in M. Migliori, Tra polifonia e puzzle. Esempi di rilettura del "gioco" filosofico di Platone, in La struttura del dialogo platonico, a cura di Giovanni Casertano, Loffredo, Napoli 2000, pp. 171-212. 
Platone ha inserito nei dialoghi, emergono problemi e soluzioni che altrimenti rischiano di essere ignorati, con conseguenze assai rilevanti sul piano interpretativo.

\section{Un errore volontario}

Parto da un testo che ho già sottoposto ad analisi, ma che devo nuovamente presentare perché costituisce un caso "estremo" e, come tale, capace di evidenziare la portata di quanto stiamo dicendo. Si tratta infatti di un errore voluto, ostentato e non corretto esplicitamente, ma solo implicitamente.

Il passo implicato è quello in Filebo, $33 \mathrm{C}-35 \mathrm{C}$. Socrate vuole sottolineare il ruolo determinante che ha l'anima in quanto sede dei desideri, anche di quelli "corporei", come la fame e la sete. Per raggiungere questa conclusione Platone deve svolgere una trattazione sulle forme conoscitive, facendo alcune distinzioni:

affezioni deboli che sfuggono all'anima affezioni che passano dal corpo all'anima conservazione di sensazioni abbiamo assenza di sensazione abbiamo sensazioni abbiamo memoria

Ma - prima "stranezza" - all'interno di questa lineare trattazione, Platone inserisce la distinzione tra memoria e reminiscenza, una questione che non ha, apparentemente, alcuna connessione con il tema ${ }^{12}$. Socrate stabilisce un forte stacco tra la memoria, che è puramente recettiva e passiva, e la reminiscenza, che è attiva e autonoma: si tratta di un'azione che l'anima compie da sola, un'azione forte (un "rivivere") che si esplica sia sul piano sensoriale sia su quello intellettuale.

Il successivo svolgimento porta a concludere che la sede dei desideri è l'anima perché, quando il corpo è vuoto, il desiderio chiede una cosa diversa (essere riempito) da quello che il corpo prova; l'anima agisce in forza della sensazione e della memoria.

${ }^{12}$ Filebo, 34 B 2 - C 2. 
L'esposizione è perfetta e lineare. Tuttavia, anche questo testo contiene un nuovo inserimento apparentemente inesplicabile, un brano che sembra "inutile":

SOCRATE - Che, dunque? Colui che si trova vuoto per la prima volta, potrebbe forse attingere dalla sensazione del riempimento o dalla memoria un'esperienza che non prova nel presente né mai ha provato nel passato?

PROTARCO - E come potrebbe? ${ }^{13}$

Socrate introduce la questione della "prima volta" ed esclude logicamente che in quel caso si possa far uso della memoria. Il fatto è che tale inoppugnabile affermazione contraddice la soluzione proposta alla fine del brano, che quindi risulta errata:

SOCRATE - Dunque, non rimane altra ipotesi se non che sia l'anima ad avere rapporto con il riempimento, evidentemente in virtù della memoria. Infatti, con che cos'altro potrebbe averlo?

PROTARCO - Con nient'altro, direi ${ }^{14}$.

Non c'è dubbio che qui ci troviamo di fronte ad un "errore volontario" in senso forte, proprio perché la sua evidenza è ricavata dalle parole dell'Autore e non da inferenze e ragionamenti del lettore. In caso contrario bisognerebbe dire che Platone è fuori di senno: dopo aver escluso (senza alcuna necessità argomentativa!!) che la prima volta l'anima desiderante possa ricorrere alla memoria, afferma subito dopo che l'unico strumento che può spiegare il desiderio è la memoria. Un errore così evidentemente segnalato non è plausibile. E infatti Socrate inserisce anche una doppia maliziosa sottolineatura, in quanto nella conclusione dice che "non rimane altra ipotesi» possibile e chiede «con che cos'altro potrebbe averlo?».

${ }^{13}$ Filebo, 35 A 6-10.

${ }^{14}$ Filebo, 35 B 11 - C 2. 
Il fatto è che Platone, fedele all'impegno didattico che abbiamo sopra illustrato, ha già fornito nel testo l'indicazione che consente al lettore che ha seguito il gioco platonico di trovare la soluzione. Chi legge il testo "facendo filosofia" ricorda l'inutile trattazione sulla reminiscenza. Se c'è una funzione attiva dell'anima, c'è un'altra possibile risposta: alla base del desiderio c'è la reminiscenza, non la memoria. Il che costituisce, anche teoreticamente, una risposta di ben diversa rilevanza.

Come ho detto, non è importante, in questa sede, accettare questa soluzione: bisogna però riconoscere che qui ci troviamo di fronte ad un gioco estremo e che, se non si legge il testo con quest'ottica, ci si trova di fronte ad un passo assurdo. Si può, ovviamente, rifiutare l'ipotesi proposta, ma solo per proporne un'altra che dia ragione di questo evidente "gioco" filosofico.

\section{Una maturità precoce?}

Siamo partiti, per così dire, dalla fine, da un brano "tardo" ed estremo e, proprio per questo, spero, chiarificatore. Volendo procedere ora in modo più graduale, per evidenziare la portata di questa proposta metodica potremmo cominciare ad applicarla alla stessa questione dei "giochi" platonici.

Molti di coloro che sono propensi ad accettare questo ambito di riflessione pensano poi che esso vada limitato ai dialoghi tardi o, al massimo, a quelli di mezzo, in quanto sarebbe frutto di una maturazione che il maestro dell' Accademia avrebbe poi esplicitato prima nel Fedro, poi nella Lettera Settima. In realtà, se assumiamo correttamente la questione (si tratta - ribadiamo - di una impostazione educativa connessa ad una concezione della filosofia), troviamo evidenze di tale impostazione fin dai primi dialoghi. Ciò costituisce anche una spiegazione della loro "aporeticità".

La testimonianza più evidente la incontriamo in uno dei primissimi dialoghi, l'Eutifrone: l'Autore mostra di conoscere la soluzione, che però non viene fornita al lettore.

Socrate ed Eutifrone discutono della santità, a partire 
dall'accusa che quest'ultimo muove al padre, reo di aver ucciso un colono. Dopo un primo infelice esempio di santità, Zeus che incatena Crono, un mito che Socrate esplicitamente dichiara di non poter credere, Eutifrone propone una prima definizione: santo è ciò che è caro agli Dei. In questo modo egli cerca di rimanere all'interno della tradizione e di valorizzare tutti quei rituali con cui gli uomini credono di far cose gradite alle divinità. Si precisa poi che devono essere gradite a tutti gli dei, ma la risposta risulta insufficiente. Socrate ha buon gioco a mostrare che, mentre l'essere amato è una caratteristica estrinseca, la santità è una qualità intrinseca di un oggetto o di una azione. Ciò porta a concludere che una realtà, che è in sé santa, viene poi, proprio per questo, amata dagli dei.

Socrate stesso, a questo punto, cerca di far uscire la discussione dall'impasse ponendo la questione del rapporto tra giusto, concetto più ampio, e santo: si tratta di capire quale parte del giusto sia il santo.

Si opera una diairesi: una parte del giusto si prende cura degli uomini, un'altra parte degli dei ed è questa la santità. Occorre vedere di che tipo di cura si parli. Socrate osserva che non può essere simile a quella dell'allevatore di animali, ma a quella del servitore nei confronti del padrone. Ma anche così non si è ancora detto quale effetto produca tale cura: bisogna determinare il servizio, il tipo di collaborazione che gli uomini possono rendere agli dei. Per questo bisogna capire che cosa gli dei stessi si propongono:

SOCRATE: E che diremo, allora, dei fini molteplici e belli che gli dèi perseguono? Quale è lo scopo principale che essi perseguono?

EUTIFRONE: Te lo dissi anche poco fa, o Socrate, che occorre un notevole impegno, per poter imparare con esattezza come stiano queste cose. Tuttavia, ti dirò in breve questo: che, se uno è capace di dire e di fare cose gradite agli dèi, pregando e sacrificando, queste sono azioni sante; e siffatte azioni sono quelle che salvano le famiglie e le città. Invece, le azioni 
contrarie a quelle gradite agli dèi sono empie: e sono queste che sovvertono e rovinano ogni cosa ${ }^{15}$.

La risposta di Eutifrone risulta particolarmente inadeguata anche perché, come Eutifrone stesso sottolinea, ritorna alla definizione precedente che identificava santità con "gradito agli dei". Ciò potrebbe solo confermare il basso profilo teoretico di questo personaggio se non fosse per lo sconcertante commento di Socrate:

Avresti potuto dirmi molto più brevemente, o Eutifrone, se avessi voluto, ciò che io ti ho domandato, ma è evidente che non sei disposto ad istruirmi. Infatti, anche ora, proprio mentre eri sul punto di farlo, ti sei ritirato. Se me l'avessi detto, avrei ormai imparato da te che cosa sia la santità. $\mathrm{E}$ ora, poiché è necessario che l'amante segua l'amato dovunque questi lo conduca, daccapo, dimmi...» ${ }^{16}$.

Quello che deve colpirci è che il filosofo, evidentemente, sa qual è la risposta che Eutifrone gli nega, altrimenti non potrebbe ripetutamente affermare che questi si è trattenuto e che, se non l'avesse fatto, egli avrebbe "appreso" che cos'è la santità. Ora, a parte il gioco di "sapere e non sapere", questa situazione non ha senso nel confronto in atto: Socrate potrebbe dare lui la risposta, visto che prima ha impostato la questione del rapporto con il giusto. Invece rinuncia e quindi il dialogo si avvia alla sua conclusione aporetica. Ma tale esito è determinato da questa errata risposta di Eutifrone non corretta da Socrate.

Ora, se usciamo dalla finzione scenica e ci poniamo di fronte all'autore che ha costruito tale finzione, se cioè leggiamo questo dialogo come un messaggio filosofico rivolto a noi, vediamo che Platone ha messo in bocca ad Eutifrone una risposta inadeguata ed ha segnalato con forza tale inadeguatezza. L'Autore ci sta

${ }^{15}$ Eutifrone 14 A 9 - B 7.

${ }^{16}$ Eutifrone 14 B 8 - C 5. 
dicendo che qui va posta una risposta diversa e che tale risposta è la soluzione del problema impostato nel dialogo. Ma tale esito resta "non detto" per volontà di Platone che conduce il dialogo all'esito aporetico: è il lettore che deve andare alla ricerca nel dialogo di quelle allusioni che lo aiutano a formulare la risposta ${ }^{17}$. Come si vede, siamo di fronte alla struttura formale di un rebus: la soluzione c'è, si indica anche il "luogo" teorico" in cui andrebbe collocata, ma non è scritta: va trovata.

Ora, questo atteggiamento, perfettamente coerente con quanto siamo venuti dicendo nelle premesse di metodo, ci porta ad una riflessione su "chi" ha scritto un simile dialogo: si tratta evidentemente di un "maestro" che ha già elaborato una propria impostazione educativa rigorosa e che si ritiene autorizzato a "sfidare" il lettore, è un autore maturo che manifesta un'evidente sapienza nella costruzione architettonica del dialogo, semplice in apparenza, ma estremamente studiato nelle movenze e nell'atteggiamento di Socrate. Ci troviamo, quindi, di fronte ad un quadro contrario a quello tradizionale che, come in molti casi analoghi, giudica questo dialogo "giovanile" sulla base di una relazione stretta tra forma del dialogo (cioè semplicità espositiva e contenuto elementare) da una parte ed età dell' Autore dall'altra. Ma se vogliamo conservare questa relazione, dobbiamo dire che l'Autore di questo gioco rilevante e complesso è un uomo maturo e un maestro affermato. E quindi chiederci: ci sono dialoghi "giovanili" di Platone?

${ }^{17}$ Cfr. G. Reale, in AA. VV., Platone, Tutti gli scritti, a cura di G. Reale, Rusconi, Milano 1991, $1994^{4}$ (traduzioni di M. L. Gatti, M. T. Liminta, C. Mazzarelli, M. Migliori, R. Radice, G. Reale), p. 19-20, n. 29: in questo caso dobbiamo affermare che l'Autore «volutamente, non rende esplicita la risposta, che il lettore deve saper trarre da solo... Platone darà, peraltro, una chiara e inequivoca indicazione allusiva a p. $15 \mathrm{~A}$, in modo che il lettore attento non esca di strada insieme ad Eutifrone».

${ }^{18}$ Ad esempio, la distinzione tra cura, qui $\theta \varepsilon \rho \alpha \pi \varepsilon i \alpha$, e all'allevamento degli animali, costituisce il fulcro della correzione della prima diairesi del Politico, 275 D -276 D, e quindi caratterizza l'atteggiamento del politico verso i cittadini.

${ }^{19}$ Un esempio ancora più evidente si trova in Gorgia, $463 \mathrm{~A}-466 \mathrm{~A}$. 
Questa redazione "non giovanile" del dialogo trova una serie di ulteriori conferme che qui non possiamo approfondire. Tralasciando argomenti specifici ${ }^{18}$, non dovrebbe sfuggire che ci troviamo di fronte ad una prima diairesi, cioè all'emergere del metodo che solitamente viene riservato al Platone maturo ${ }^{19}$ e che con quest'opera "aporetica" Platone imposta precocemente uno dei problemi di fondo della sua filosofia, il rapporto con Dio, tema che ricorre costantemente nella sua produzione fin nella sua ultima opera $^{20}$.

\section{Il rinvio della trattazione del Bene}

Se su un tema come quello della santità Platone pratica la tecnica del rinvio e dell'allusione, non può che risultare ovvia la scelta di operare con altrettanta e anche maggiore spregiudicatezza a proposito del Bene. Qui saremo sufficientemente sommari, perché si tratta di testi molto conosciuti e studiati ${ }^{21}$. Val la pena analizzarli, tuttavia, perché anche in questo caso non abbiamo solo il rinvio: Platone da una parte mostra di sapere, dall'altra ostenta di non voler dire, eppure dice e molto, solo che questo "molto" deve essere scoperto dal lettore stesso.

I dialoghi di riferimento sono, com'è noto, soprattutto due: Repubblica e Filebo.

Nella Repubblica, Platone si rifiuta esplicitamente di parlare del Bene, limitandosi a riferire sul «figlio del Bene e somigliantissimo a lui ${ }^{22}$, non pagando il debito ma i soli "interessi" ${ }^{23}$ : tutto viene ricondotto ad una serie di paralleli con il sole.

Ora, tralasciando le ragioni con cui Socrate stesso giustifica

${ }^{20} \mathrm{Cfr}$. ad esempio, Leggi, IV, $716 \mathrm{C}-717 \mathrm{~B}$.

${ }^{21} \mathrm{Su}$ questo tema cfr. quanto dico in: Sul Bene. Materiali per una lettura unitaria dei dialoghi e delle testimonianze indirette, in New Images of Plato, Dialogues on the Idea of the Good, Edited by G. Reale and S. Scolnicov, Academia Verlag, Sankt Augustin 2002, pp. 115-149

${ }^{22}$ Repubblica, VI, 506 E 3-4.

${ }^{23}$ Repubblica, VI, 507 A 2.

Educ. e Filos., Uberlândia, v. 20, n. 40, p. 41-80, jul./dez. 2006. 
questa rinuncia ${ }^{24}$, lo stesso fatto di affermare e poi svolgere un rapporto di somiglianza così radicale implica una conoscenza approfondita. Inoltre, si afferma che Adimanto ha già sentito Socrate parlare di questo tema più volte ${ }^{25}$. In tal modo la rinuncia a "scriverne" risulta particolarmente evidenziata. Infatti, se restiamo all' interno della fiction, Socrate non dovrebbe avere alcuna difficoltà, dal punto di vista della comunicazione interna al dialogo, a richiamare in breve le affermazioni che Adimanto ha già sentito senza aggiungervi ulteriori spiegazioni, invece di procedere faticosamente attraverso analogie e allegorie, con spiegazioni annesse e riferimenti incrociati.

Tale situazione paradossale si spiega solo se si ricorda che la discussione orale c'è solo nell'immagine fittizia, perché nella realtà noi siamo di fronte ad uno scritto. In questo caso scattano i vincoli che il filosofo scrittore si è autoimposto per ragioni filosoficoeducative. Se ragioniamo in termini di teatro filosofico, di quello che la scena dice a noi e di quello che ci porta a sentire e a pensare, vediamo come Platone "parlando" con il lettore comunica di non volere esplicitare la "definizione" del Bene.

Per avere la conferma che Platone ha architettato uno dei suoi giochi, basta che il lettore raccolga le caratteristiche del Bene che emergono "malgrado" questa rinuncia:

1. scienza e verità sono belle, ma non sono il Bene, che è diverso e ancor più bello; pertanto, la sua condizione deve essere giudicata superiore $^{26}$;

2. il Bene è causa di conoscenza e verità ed è di conseguenza conoscibile ${ }^{27}$;

3. il Bene fornisce alle realtà non solo la conoscibilità, ma anche l'essere e la sostanza;

${ }^{24}$ Il tema è lasciato da parte "ora" ( ò̀ vôv, 506 E 1) per l'impossibilità di chiarire,

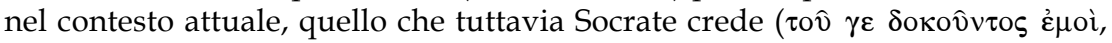
506 E 2) in proposito.

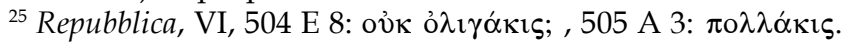

${ }^{26}$ Repubblica, VI, 509 A 4-5.

${ }^{27}$ Repubblica, VI, 508 E. 
egli stesso non è essere, ma superiore all'essere per dignità e potenza $^{28}$.

Come si vede, questa realtà non è affatto inconoscibile o sconosciuta: malgrado il rinvio Platone ci dice che abbiamo un Principio Primo il quale, pur chiamandosi Bene, non è solo causa assiologica, ma anche ontologica e gnoseologica.

La stessa situazione si ripresenta, ad un livello di maggiore complessità, ma anche di maggiore chiarificazione, nel Filebo ${ }^{29}$. Di nuovo, Platone si rifiuta di definire il Bene, pur essendo chiaro che la vita buona, tema del Filebo, va giudicata alla luce del Bene: infatti, la scelta fatta all'inizio a favore della vita mista si basa sui caratteri formali del Bene ${ }^{30}$; inoltre, il criterio con cui assegnare il "secondo posto", tema che giustifica la continuazione del dialogo, è l'affinità di uno dei due contendenti, pensiero e piaceri, alla causa, a ciò che rende buona la vita mista ${ }^{31}$. Pertanto, non stupisce che si debba "cogliere il Bene, o chiaramente per sé o anche qualche sua traccia $»^{32}$ e che nella mescolanza più bella e più stabile si possa comprendere «che cosa sia per natura, nell'uomo e nel tutto, il Bene e quale Idea bisogna in qualche modo attribuirgli» ${ }^{33}$.

Tuttavia, come nella Repubblica, giunto al momento di definire in qualche modo il Bene, Socrate dichiara che si comporterà come

${ }^{28}$ Repubblica, VI, 509 B 7-10.

${ }^{29}$ Possiamo, in questo caso, rinviare ai nostri studi analitici: M. Migliori, L'uomo tra piacere, intelligenza e Bene. Commentario storico-filosofico al "Filebo" di Platone, Vita e Pensiero, Milano 1993; 19982; Lo sviluppo tempestoso di un gioco compatto: la struttura del Filebo, in Il Filebo di Platone e la sua fortuna, a cura di P. Cosenza, M. D'Auria Editore, Napoli 1996, pp. 353-373; L'Idea del Bene nel Filebo, in AA. VV., Studi in onore di Antonio Possenti, a cura di G. Almanza Ciotti, S. Baldoncini, G. Mastrangelo Latini, Istituti Editoriali Poligrafici Internazionali, Pisa-Roma, Macerata 1998, pp. 429-457.

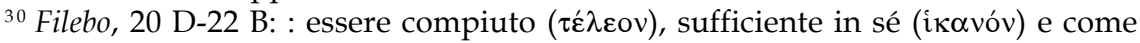

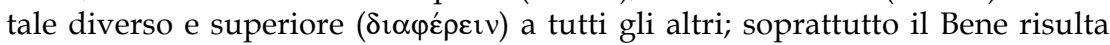

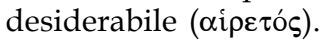

${ }^{31}$ Filebo, 22 C-E; le stesse affermazioni vengono poi ribadite nel momento in cui inizia la trattazione finale $(60 \mathrm{~B}-\mathrm{C}, 61 \mathrm{~A})$.

${ }^{32}$ Filebo, 61 A 4-5.

${ }^{33}$ Filebo, 64 A 1-3. 
chi, cercando una determinata persona, si informa sulla sua casa per poterlo poi incontrare ${ }^{34}$; in seguito, giunto nei "vestiboli" della casa del Bene, si limita a dire che la causa che rende apprezzabile qualsiasi mescolanza è quella che realizza misura e proporzione, cioè il bello e la virtù ${ }^{35}$, parla cioè dei soli effetti.

Tuttavia, queste stesse affermazioni confermano che il Bene è conoscibile è conosciuto, anzi, nel suo solito modo allusivo, Platone ci dà tre indicazioni molto forti sulla natura del Bene:

1. il Bene è "potente" ( $\dot{\eta} \tau o \hat{v} \alpha \dot{\alpha} \gamma \alpha \theta 0 \hat{v} \delta v ́ v \alpha \mu \imath \varsigma, 64$ E 5) a

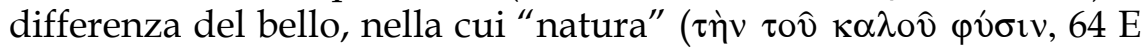
6) pure si manifesta e si nasconde;

2. nell'elenco dei cinque beni che chiude il dialogo, troviamo al primo posto "ciò che è nei pressi della misura", $\pi \rho \hat{\omega} \tau o \nu \mu \varepsilon \dot{\varepsilon} \nu \pi \eta$ $\pi \varepsilon \rho \grave{~} \mu \varepsilon \varepsilon^{\prime} \rho \nu^{36}$. La misura in quanto tale, su cui tanto ci si è soffermati in precedenza, non è in questo elenco, per cui o supponiamo che non sia un bene o dobbiamo pensare che sia il Bene stesso, la causa che, come tale, non può essere nell' elenco dei "beni". Questo risulta confermato dal primato che ha, tra i beni, ciò che è "nei pressi" della misura, mentre gli altri elementi cui la misura è stata prima costantemente associata, proporzionato, bello, completo e sufficiente, sono relegati al secondo posto ${ }^{37}$;

3. Socrate afferma:

Dunque, se non possiamo cogliere il Bene in una sola Idea, dopo averlo colto con tre, ossia bellezza, proporzione e verità, diciamo che attribuiamo giustamente a questo, preso come un uno, la causa delle realtà che sono nella mescolanza, e che la bontà della mescolanza deriva da questo, in quanto esso è Bene ${ }^{38}$.

\footnotetext{
${ }^{34}$ Filebo, 61 A-B.

${ }^{35}$ Filebo, 64 D-E.

${ }^{36}$ Filebo, 66 A 6.

${ }^{37}$ Filebo, 66 B 1-2.

${ }^{38}$ Filebo, 65 A $1-5$.
} 
Quindi, c'è un principio Uno che sintetizza in sé gli elementi che troviamo in bellezza, proporzione e verità, che è causa ontologica delle realtà che troviamo nella mescolanza e, in quanto Bene, causa della bontà della mescolanza stessa; inoltre, vista la presenza della verità, è anche causa gnoseologica.

Questa affermazione, molto esplicita, trova la sua conferma in un gioco che caratterizza l'intera struttura del dialogo. Com'è noto, nella prima parte Platone svolge una lunga trattazione metafisica, in cui descrive la realtà come un misto uni-molteplice, che dipende dall'azione di un Peras su un Apeiron. Tale trattazione sembra quasi inutile a molti studiosi e scompare del tutto nella seconda parte del dialogo.

In realtà il Peras svolge nella prima parte la stessa funzione che compete al Bene-Misura nella seconda:

a) è una potenza che agisce sull'indeterminato, un limitante che rende possibile l'esistenza stessa del misto in tutti gli aspetti della realtà, dalle cose concrete alla musica, alle stagioni fino alle molte cose belle che esistono nell' anima ${ }^{39}$;

b) b) ha una funzione valoriale: la Dea (causa efficiente) vede che le cose indeterminate sono cattive, che l'ordine è buono e lo realizza per l'azione del Peras $^{40}$.

A questo punto, il gioco diviene chiaro: Platone ha fatto due volte lo stesso tipo di affermazioni: Se colleghiamo le due parti del dialogo senza lasciarci fuorviare dalla differenza terminologica adottata dall'Autore, vediamo che per Platone c'è un principio limitante, polifunzionale, che agisce su un principio di infinitezza per dar luogo ad un cosmo misto in cui

$c^{\prime}$ è molto illimitato e sufficiente limite, e, al di sopra di essi, una causa non da poco, la quale, ordinando e regolando gli anni, le stagioni e i mesi, può, a buon diritto, essere chiamata sapienza e intelligenza ${ }^{41}$.

\footnotetext{
${ }^{39}$ Filebo, $25 \mathrm{D}-26 \mathrm{C}$.

${ }^{40}$ Filebo, 26 B 7 - C 1.

${ }^{41}$ Filebo, 30 C 4-7.
} 
Il Filebo, quindi, apporta molti chiarimenti sulla natura del Principio polare, in particolare sulla struttura delle cause: Dio ha funzione di causa efficiente ${ }^{42}$, l'Apeiron quella di causa materiale, il principio come Peras agisce come causa formale, confermata dalla sua funzione di ordine e dall' affermazione che (come Bene) è causa della bontà della mescolanza, mentre come Bene è anche causa finale, sottolineata dalla ridondante affermazione della sua desiderabilità.

\section{Un esercizio infinito}

Questi primi esempi dovrebbero essere sufficienti a mostrare in che senso la scrittura platonica è tesa a proporre stimoli filosofici al lettore. Questa costruzione di esercizi, certamente non fini a se stessi ma intrinsecamente filosofici, trova il suo culmine nel Parmenide, che non a caso risulta uno dei testi più equivocati. Infatti questo dialogo è centrale per giustificare la cosiddetta "crisi" del sistema platonico, una sorta di autocritica che avrebbe aperto la strada a nuovi e diversi sviluppi. Se però prestiamo attenzione ai segnali che Platone ci fornisce, il quadro cambia radicalmente ${ }^{43}$.

Si dice spesso che vi sono sette obiezioni di Parmenide, ma in realtà si tratta di tutt'altro. Subito prima, Socrate ha battuto la posizione di Zenone con l'inserimento della dottrina delle Idee. Parmenide, che approva esplicitamente l'obiezione del giovanissimo filosofo ateniese fatta contro il suo "amasio", gli chiede se però conosce i problemi che tale teoria solleva. Seguono otto questioni:

1. premessa: contrariamente a quello che sembra supporre il giovanissimo Socrate, esistono idee di tutte le cose, anche di quelle ridicole (130 B-E);

${ }^{42}$ Filebo, 26 E - 27 C; 30 C-D.

${ }^{43}$ Anche in questo caso, per ulteriori verifiche ed approfondimenti, rimando al mio studio analitico: M. Migliori, Dialettica e verità. Commentario filosofico al "Parmenide" di Platone, Vita e Pensiero, Milano 1990, 2000². 
2. prima critica di Parmenide: le cose non partecipano né di tutta l'Idea né di una parte (130 E - $131 \mathrm{E})$;

3. seconda critica di Parmenide: se dalle cose si risale all'Idea si procede all'infinito (131 E - $132 \mathrm{~B}$ );

4. prima variante proposta da Socrate e rifiutata da Parmenide: le Idee sono pensieri (132 B-C);

5. seconda variante proposta da Socrate e rifiutata da Parmenide: le cose sono copie e le idee sono modelli (132 C - 133 A);

6. prima difficoltà teorica maggiore, conseguente alla rigida separazione tra mondo e Idee: l'uomo non avrà scienza (133 B - 134 C);

7. seconda difficoltà teorica maggiore, conseguente alla rigida separazione tra mondo e Idee: Dio avrebbe la massima conoscenza e il massimo potere, ma non conoscerebbe né avrebbe potere su questo mondo (134 C-E);

8. Conclusione di Parmenide: tuttavia non si può fare a meno delle Idee in funzione della dialettica, cioè della filosofia (134 E - $135 \mathrm{C})$.

Si noti l'architettonica del brano: la struttura delle "critiche" ha una duplice articolazione, quelle "tecniche" (2 e 3) e quelle teoretiche di fondo (6 e 7) ed ha al suo interno due ipotesi, probabilmente accademiche, che cercano di aggirare le difficoltà con una diversa formulazione della teoria delle Idee (4 e 5), ma cosa soprattutto rilevante ai fini del nostro discorso - sono poste all'interno di due affermazioni perentorie a favore dell'esistenza delle Idee (1 e 8).

Come si vede, lo stesso schema chiarisce che qui non si sta affatto attaccando la teoria delle idee: all'inizio e alla fine si afferma con forza la necessità di questo strumento teorico. Platone sa tanto bene che è possibile fraintendere questo intervento che alla fine esplicita la sua posizione in modo reiterato: il vero filosofo scopre le Idee in quanto senza non si dà filosofia. Infatti queste difficoltà spingono molti a sostenere che le Idee

non esistono o che, se esistono, necessariamente sono inconoscibili alla natura umana; così dicendo sembrerà dire 
una cosa sensata e, come osservavamo prima, sarà difficile convincerlo. Solo un uomo molto dotato potrà comprendere che esiste un genere per ogni realtà e una sostanza in sé e per sé; solo uno ancora più eccezionale saprà scoprirlo e potrà essere capace di insegnarlo ad un altro, dopo averlo esaminato in tutti gli aspetti. Sono d'accordo con te, Parmenide - confermò Socrate. - Dici esattamente quello che penso.

Però, Socrate - disse Parmenide - se qualcuno non vorrà ammettere che esistano le Idee delle realtà, a causa di tutte le difficoltà già dette e di altre ancora, e non vorrà porre un'Idea per ogni singola realtà, non avrà un punto di riferimento per il suo pensiero, in quanto non ammetterà un'Idea di ciascuna delle realtà che esistono, che sia sempre la stessa per ciascuna realtà: così distruggerà la forza della dialettica. Ma di un tale problema mi sembra che tu abbia già avuto particolare sentore.

Dici il vero- riconobbe.

Che cosa farai dunque della filosofia? Dove ti volgerai, se non hai una soluzione per questi problemi?.

Non mi sembra di vederne alcuna all'orizzonte.

Infatti, Socrate - disse - tu hai tentato di definire il bello, il giusto, il buono e ogni singola Idea troppo presto, prima di esserti esercitato adeguatamente. Infatti, l'ho capito l'altro giorno, ascoltandoti qui mentre discutevi con il nostro Aristotele. Bello e divino, sappilo, è lo slancio che ti spinge verso gli argomenti. Ma, visto che sei giovane, esercitati, impegnandoti a fondo in quell'attività che può sembrare inutile e che $i$ più considerano puro gioco di parole, altrimenti la verità ti sfuggirà. ${ }^{44}$.

Il problema è che per salvare le Idee occorre fare un lungo esercizio dialettico, tanto complesso che Socrate lo definisce «esercizio senza fine ${ }^{45}$ e che così Parmenide presenta:

${ }^{4}$ Parmenide, 135 A 4 - D 6.

${ }^{45}$ Parmenide, 136 C 6. 
In breve, posto che di un qualsiasi oggetto si ipotizzi sempre l'esistenza, la non esistenza e una qualsiasi altra affezione, devi esaminare le conseguenze sia in rapporto all'oggetto stesso, sia in rapporto a ciascuno degli altri oggetti singolarmente considerato, qualunque tu scelga, sia in rapporto a più oggetti intrecciati tra loro, sia in rapporto a tutti gli oggetti presi insieme. Inoltre devi esaminare anche gli altri, sia rispetto a se stessi, sia rispetto a qualsiasi altro oggetto tu voglia scegliere, con l'ipotesi che esista o che non esista, se vuoi vedere con sicurezza la verità dopo aver svolto compiutamente il tuo esercizio ${ }^{46}$.

Il giovane filosofo non riesce a comprendere bene la proposta del vecchio Eleate, per cui Parmenide è "costretto" dalle insistenti preghiere dei presenti a offrirne un piccolo esempio nella seconda parte del dialogo, esempio di un esercizio che è quindi esso stesso un esercizio. Ma di filosofia, quindi pieno di giochi, di problemi, di indicazioni a tutto campo sulla metafisica e sulla protologia platonica. Ma non è questo il luogo per approfondirli.

Resta però chiaro che una lettura diversa delle "critiche" di Parmenide rende inesplicabile la stessa struttura del dialogo che passa dal superamento della posizione dei fisici (qui rappresentati da Zenone) fino al chiarimento della natura dialettica del mondo delle Idee, secondo un modello che era stato anticipato formalmente nel Fedone ${ }^{47}$.

${ }^{46}$ Parmenide, 136 B 6 - C 5.

${ }^{47}$ Ho già mostrato (M. Migliori, Il "Parmenide" e le dottrine non scritte di Platone, Istituto Suor Orsola Benincasa, Napoli 1991 (pp. 117), ora anche in Verso una nuova immagine di Platone, a cura di G. Reale, Vita e Pensiero, Milano 1994², pp. 165-222, pp. 169-184) come una serie di parallelismi forti sottolineino il già evidente collegamento tra lo svolgimento del Parmenide e la presentazione delle Idee e del loro possibile "superamento" in una dimensione protologica presente nel Fedone, $95 \mathrm{E}$ - $107 \mathrm{~B}$ : ciò che in questo ultimo dialogo è presentato come possibilità e risolto su un piano puramente formale, viene poi svolto (in modo chiaro ed esplicito, anche se sempre non adeguato né compiuto) nel Parmenide. 


\section{Una necessaria diffidenza}

Se Platone scrive utilizzando la tecnica che stiamo cercando di illustrare, il lettore è obbligato ad essere sempre cauto e, per così dire, sospettoso anche laddove il testo sembra evidente. Propongo a questo proposito un altro esempio in qualche misura estremo.

Se si chiedesse ad uno studioso qual è la definizione di sofista che Platone propone difficilmente non si sarebbe d'accordo su questa:

l'imitare l'arte che produce contraddizioni, parte simulatrice dell'arte di produrre opinioni, del genere che produce apparenze sulla base della capacità di produrre immagini, sezione non divina ma umana dell'attività produttiva, cioè quella parte che fa meraviglie nei discorsi ${ }^{48}$

visto che il testo stesso afferma

chi dirà che "di questa stirpe e di questo sangue" è il vero sofista, dirà, come sembra, la cosa più vera ${ }^{49}$.

Eppure ci apprestiamo a mostrare che non è così e che questa indubitabile verità va tuttavia integrata con una serie di dati, sulla base di chiarissime indicazioni fornite da Platone stesso.

\section{La struttura di definizioni del "Sofista"}

La prima questione riguarda la serie di altre definizioni che riempiono la prima parte del dialogo. In breve $\mathrm{e}^{50}$, abbiamo 4 diairesi e sette definizioni: la prima diairesi $(221$ C - 223 B) ci fornisce la

${ }^{48}$ Sofista, 268 C 8 - D 2.

${ }^{49}$ Sofista, 268 D 2-4.

${ }^{50}$ Per una più esatta comprensione della nostra ricostruzione del Sofista, rinviamo a un nostro articolo: M. Migliori, Verso il filosofo: dialettica e ontologia nel "Sofista" di Platone, "Rivista di filosofia neo-scolastica», 91 (1999), pp. 171-204, e al volume Il "Sofista" di Platone, Valore e limiti dell'ontologia, Morcelliana, Brescia 2006. 
prima definizione: il sofista è un cacciatore di giovani ricchi; la seconda diairesi (223 C - $224 \mathrm{E})$ ci dà quattro definizioni, che sono varianti del sofista commerciante e produttore di conoscenze che riguardano l'anima, sia proprie sia altrui, sia al minuto sia "all'ingrosso"; con la terza diairesi (224 E - 226 A) incontriamo la sesta definizione: il sofista è un erista; la quarta diairesi (226 B - 231 B) ci fornisce la settima definizione: un sofista di nobile stirpe, purificatore dell'anima.

Ora, non solo queste definizioni non vengono mai in nessun modo negate o anche solo messe in discussione, ma

a) trovano la loro giustificazione nella natura multiforme dell'oggetto indagato, su cui Platone molto insiste ${ }^{51}$;

b) costituiscono la base per la successiva indagine tesa a trovare una definizione che in qualche modo le unifichi ${ }^{52}$.

L'ultima definizione è tuttavia radicalmente diversa da quelle precedenti proprio per la struttura diairetica della definizione. Infatti, la diairesi inizia distinguendo, nell'ambito dell'attività $(\tau \varepsilon ́ \chi \vee \eta)$, quella produttiva e quella acquisitiva: mentre le prime definizioni sono tutte acquisitive, quella finale è produttiva. Si tratta quindi di una differenza radicale. Per cercare di capire questa modificazione, dobbiamo prestare attenzione a due dati.

Il primo è costituito dalla quarta diairesi che ha un andamento del tutto diverso: a prima vista non possiamo porla in nessuna delle due articolazioni proposte, perché è così strutturata:

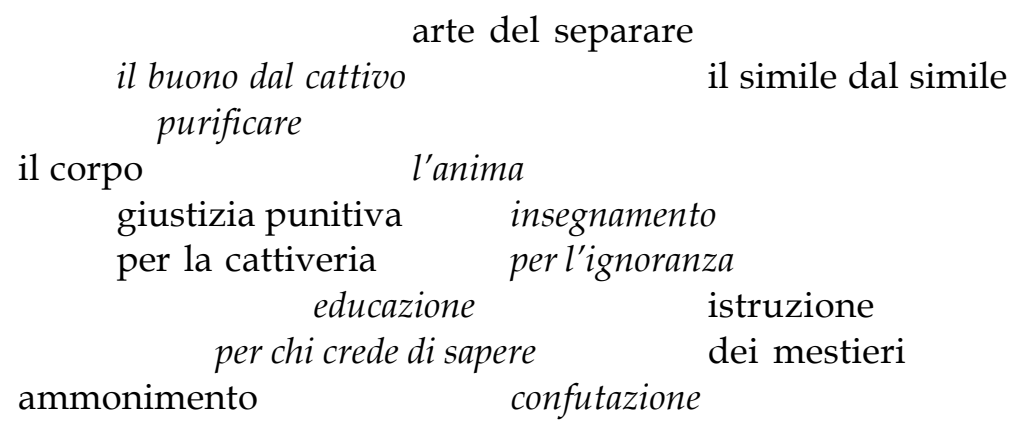

51 Sofista, 218 C-D; 223 C; 225 E; 226 A; 231 C; 236 D; 240 C; 261 A.
52 Sofista, 231 C - 232 B.

Educ. e Filos., Uberlândia, v. 20, n. 40, p. 41-80, jul./dez. 2006. 
Platone non ci dice affatto come va intesa quest'arte del separare da cui parte questa diairesi, che quindi risulta già per questo aspetto "straordinaria". Molto più rilevante è poi l'imbarazzo che Platone mostra nel collocare questa figura tra gli altri sofisti. In effetti questi "sofisti" cercano di far superare ai loro allievi una forma di ignoranza, quella

forma ben distinta, grande e pericolosa, che si contrappone a tutte le altre... il credere di sapere qualcosa senza saperlo ${ }^{53}$.

Essi ritengono che chi è ignorante lo sia involontariamente $\mathrm{e}$ che bisogna preventivamente convincerlo della propria ignoranza, non con l'ammonimento paterno, ma con un metodo confutatorio, ponendo domande e mostrando che le risposte sono tra di loro in contraddizione.

Per tutte queste ragioni, Teeteto, dobbiamo affermare che la confutazione è la più grande e la più efficace delle purificazioni e dobbiamo anche pensare che chi non è stato confutato, fosse anche il Gran Re, proprio in quanto non è stato purificato nelle cose più grandi, è privo di educazione e brutto, proprio in ciò in cui, a chi intende essere veramente felice, converrebbe essere puro e bello al massimo grado ${ }^{54}$.

Proprio perché si tratta di un'attività educativa del tutto positiva, che opera una "purificazione" necessaria e lodevole, lo Straniero di Elea teme di attribuire ai sofisti un onore troppo grande riferendo a loro quest'arte e sottolinea che occorre prestare grande attenzione perché le somiglianze ingannano ${ }^{55}$ : siamo di fronte ad una relazione che ricorda quella tra cane e lupo, tra l'animale più selvaggio e quello più domestico ${ }^{56}$. Con tutte queste cautele, lo

${ }^{53}$ Sofista, 229 C 1-5.

${ }^{54}$ Sofista, 230 D 6 - E 3.

${ }^{55}$ Sofista, 231 A-B.

56 Sofista, 231 A. 
Straniero accetta una tale figura di sofista di nobile stirpe ${ }^{57}$, vero educatore che mira a creare le condizioni per la conoscenza e la virtù e che purifica i suoi allievi con la scoperta del loro "non sapere".

Il gioco è ora chiaro: siamo di fronte a Socrate, sofista del tutto eccezionale. Ma proprio il fatto che l'Autore ha sentito la necessità, "sofferta", di introdurre anche questo personaggio, ci deve far supporre che, in questa prima parte, Platone intenda presentarci una tipologia, in qualche misura completa e al suo tempo riconoscibile, dei sofisti che avevano operato in Grecia. Allora la prima parte non è "sbagliata", ma rappresenta la ricostruzione dei "tipi" di sofista come storicamente si presentavano, al livello della loro autoconsapevolezza, per quello che il pensiero comune poteva "fenomenologicamente" riconoscere e capire.

Ciò spiega il secondo dato con cui dobbiamo fare i conti. Dovendo cercare che cosa c'è a fondamento di questa figura, per arrivare alla ultima e diversissima definizione, lo Straniero deve passare attraverso il "parricidio", cioè una lunga trattazione teoretica, un passaggio obbligato del platonismo, che modifica radicalmente tutti i termini in gioco. Solo in tale visione platonica è possibile dare senso a quella definizione, che quindi svela il sofista come solo il Filosofo può pensarlo e dirlo. In senso forte solo questa è vera, anche se le altre non sono false, ma solo fenomenologiche, descrittivamente valide.

\section{Il sofista nel "Politico"}

Tuttavia, neanche questo basta, perché l'ultimo e definitivo chiarimento sul sofista può essere dato solo nel Politico. ${ }^{58}$. Questo dialogo, infatti, è posto in una condizione "straordinaria: se prendiamo la fine del Sofista e l'inizio del Politico vediamo subito

57 Sofista, 231 B.

${ }^{58}$ Anche in questo caso, per giustificare le affermazioni che farò, rimando al mio studio analitico: M. Migliori, Arte politica e metretica assiologica. Commentario storico-filosofico al "Politico" di Platone, Vita e Pensiero, Milano 1996. 
che non c'è alcuna soluzione di continuità: i due dialoghi sono in realtà anche uno solo! Così Platone ci ha segnalato che sono due i temi da trattare, e ben diversi, ma che la loro trattazione è profondamente intrecciata.

Infatti, al vertice degli avversari del vero politico, dei "falsi politici", ecco avanzare una folla differenziata di esseri molto strani, un insieme assai variegato di bestie, leoni, centauri, moltissimi satiri, bestie deboli e astute e altri mostri che rapidamente si scambiano fra di loro nature e potere ${ }^{59}$. Lo Straniero ha un moto di sorpresa, perché "solo ora" ha capito che costoro sono i sofisti.

$\mathrm{Ma}$ in realtà neanche questo è vero. Torniamo all'ultima diairesi del Sofista: possiamo farlo tranquillamente proprio perché i due dialoghi sono esplicitamente connessi, e dobbiamo farlo, se intendiamo usare questi testi per "fare filosofia". Guardiamo qual è l'ultima movenza della diairesi:

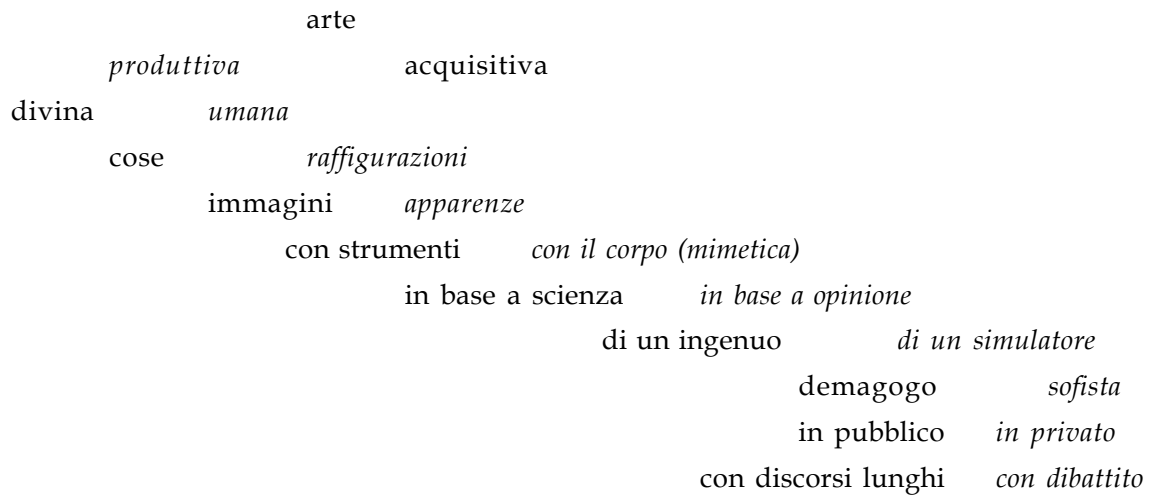

Come si vede, Platone ci aveva già detto che c'è un simulatore che ha le stesse caratteristiche del sofista, solo che opera in pubblico e con discorsi lunghi: il demagogo, quello che poi si rivelerà come il sofista-politico. Tuttavia, l'Autore non ha nemmeno fatto un gioco superfluo e la sorpresa dello Straniero è, dal punto di vista della

${ }^{59}$ Politico, 291 A-C. 
fiction, corretta. Il dato viene esplicitato alla fine del Politico, quando si torna su questi personaggi ${ }^{60}$ che cercano di svolgere attività politica senza possedere scienza, uomini di parte, che propagano le più grandi riproduzioni, anzi, sono essi stessi riproduzioni, essendo grandissimi imitatori e ciarlatani. Costoro sono definiti " $\mathrm{i}$ sofisti più grandi tra i sofisti". Ecco la ragione vera della sorpresa!

Abbiamo quindi un'ottava definizione di sofista: il sofista è il politico che opera su base non scientifica, ingannando e utilizzando la sua abilità per nascondere gli errori. Questo è l'aspetto più micidiale della sofistica, che solo qui poteva essere chiarito perché sarebbe risultato poco comprensibile senza la delineazione del vero politico.

In sintesi, esistono sei "descrizioni" adeguate e due definizioni filosofiche del sofista, una nel Sofista e una nel Politico.

\section{L'architettonica di un dialogo}

Possiamo ora offrire alcuni esempi più tecnici della pratica platonica, scusandoci in anticipo per la sommarietà della trattazione che, come già detto, vuole essere puramente "indicativa".

Il primo esempio vuole richiamare l'attenzione sull' architettonica del dialogo, che è spesso un complesso gioco di indicazioni in positivo e in negativo, di richiami e di opposizioni. Per questo può essere utile riflettere sulla particolare struttura dei discorsi in onore di Eros che troviamo nel Simposio, tralasciando le parti narrative, che pure hanno una rilevanza eccezionale.

Lo svolgimento dei vari argomenti sembra facilmente identificabile alla luce del giudizio formulato da Socrate stesso che distingue tra il suo parlare in nome della verità e l'intervento di tutti gli altri che hanno pensato solo a fare un bel discorso ${ }^{61}$. Lo stacco è decisivo e non può essere relativizzato. Il lettore può quindi:

\footnotetext{
${ }^{60}$ Politico, 303 B-C.

${ }^{61}$ Simposio, 198 D - 199 B.
} 
1. essere convinto che abbiamo una serie di discorsi falsi cui si contrappone un discorso vero;

2. essere in dubbio sulla successione dei primi discorsi sia perché stabilire l'ordine di discorsi falsi sembra scarsamente rilevante, sia perché, in assenza di indicazioni testuali, tutto si riconduce alla "fantasia" degli interpreti.

In realtà, Platone dà segnali chiari su entrambi i terreni.

Subito dopo aver detto che il solo discorso di Socrate è vero, sentiamo Dotima spiegare a Socrate che Eros non è né bello né buono. Questo però non autorizza affatto a dire che è brutto e cattivo. Per spiegare tale affermazione, senza alcuna apparente ragione, Diotima allarga il discorso alla conoscenza. C'è qualcosa di intermedio fra sapienza ed ignoranza, l'opinare rettamente senza essere in grado di fornire spiegazioni: non è un sapere, visto che non sa spiegare, ma non è neppure ignoranza, visto che coglie la realtà ${ }^{6}$.

Platone ci ha così detto che non è corretto dedurre da una verità, il discorso di Socrate, la falsità degli altri. In effetti, se fossero tutti falsi, Platone si sarebbe assunto la responsabilità di dar voce all'errore senza contraddirlo adeguatamente. Così, invece, siamo ora in grado di avvederci del fatto che ci sono solo due discorsi che vengono esplicitamente richiamati per essere respinti: evidentemente essi racchiudevano un errore così grave che Platone non poteva, da buon educatore, tacere. Il primo è l'affermazione di Fedro che le grandi figure mitiche, come Alcesti e Achille, hanno compiuto le loro azioni per la spinta di Eros, l'altro è il mito di Aristofane. Su entrambi l'affermazione di Diotima, che risale ad un momento "precedente" i discorsi che si tengono nel simposio in onore di Agatone, è chiarissima:

Sii pur certo, o Socrate. Infatti, se tu prendessi in considerazione il desiderio di distinguersi degli uomini, ti meraviglieresti della loro condotta irragionevole, a meno

${ }^{62}$ Simposio, $201 \mathrm{E}-202$ A. 
che tu non prendessi in considerazione le cose che ti ho detto, osservando come essi si trovino in una condizione straordinaria d'amore di diventare famosi e di acquistare gloria immortale che duri per sempre, e come proprio per questo siano pronti ad affrontare tutti i pericoli, più ancora che non per i figli, e a consumare le loro ricchezze e sostenere ogni sorta di fatiche e perfino a morire per questo. $\mathrm{O}$ tu credi, invece, che Alcesti sarebbe morta per Admeto, e Achille avrebbe seguito Patroclo nella morte, e il vostro Codro sarebbe andato a morire prima del tempo per salvare il regno ai figli, se essi non fossero stati convinti che della loro virtù sarebbe rimasta immortale la memoria, che ancora oggi noi conserviamo? Ci vuol altro! Credo proprio - soggiunse - che tutti facciano quello che fanno per la virtù immortale e per questa fama gloriosa, tanto più, quanto più valgono: infatti, essi amano l'immortalità ${ }^{63}$;

Però si sente fare un certo discorso, secondo cui quelli che amano sono coloro che cercano la loro metà. Il mio discorso dice, invece, che l'amore non è amore né della metà né dell'intero, a meno che, caro amico, essi non siano il Bene ${ }^{64}$.

Naturalmente, un'analisi approfondita del dialogo ci permetterebbe di scoprire bel altri riferimenti, ma, ai fini del nostro discorso, basta riconoscere il tipo di gioco platonico, che apre un vasto campo di ricerca: si tratta di vedere quali nessi di ripresa, di inveramento o di falsificazione, troviamo rispetto a discorsi che non sono necessariamente falsi, ma che hanno tutti bisogno di una verifica.

Ciò giustifica l'ordine che è tutt'altro che casuale. Anche su questo il segnale di Platone è inequivoco: si procede per pura collocazione di posto, ma, quando arriva il turno di Aristofane, il

\footnotetext{
${ }^{63}$ Simposio, 208 C 1 - E 1.

${ }^{64}$ Simposio, 205 D 10 - E 3.
}

Educ. e Filos., Uberlândia, v. 20, n. 40, p. 41-80, jul./dez. 2006. 
singhiozzo gli impedisce di parlare e al suo posto interviene Erissimaco. Così, con questa comica invenzione, Platone costruisce uno svolgimento che, in breve, può così essere ricostruito.

La prima posizione è quella puramente retorica di Fedro, un discorso errato e di scarsa importanza. Platone lo sottolinea con un inserimento che svaluta il discorso, che provocò una discussione che non merita di essere riferita, e, tramite il ritorno in scena di Aristodemo, costituisce una sorta di intervallo:

[Aristodemo] narrava che Fedro pronunciò all'incirca questo discorso, e che dopo quello di Fedro ce ne furono alcuni altri che egli non ricordava bene e, lasciandoli da parte, passò ad esporre il discorso di Pausania ${ }^{65}$.

Segue quello "umanistico" di Pausania, che propone un ideale elevato di amore stabile tra due adulti maschi sulla base di una distinzione tra due forme di eros, uno volgare e uno celeste. Poi, grazie all'invenzione del singhiozzo, c'è il medico Erissimaco, che si ricollega esplicitamente al discorso di Pausania, che gli pare giusto, per quanto riguarda la duplice natura di Eros, ma incompleto in quanto va applicato all' intero cosmo ${ }^{66}$. Così costruisce un quadro incentrato sull'universale armonica presenza di opposti. Ma egli stesso sottolinea che ha tralasciato molte cose e che toccherà ad Aristofane "colmare il vuoto" 67.

In effetti, dopo uno scambio di battute, il commediografo precisa che non farà un discorso burlesco e, alla fine del mito, sottolinea di aver fatto un discorso del tutto diverso da quello di Erissimaco $^{68}$. Ma la diversità non riguarda evidentemente il contenuto, visto che il medico subito afferma che il discorso gli è piaciuto $^{69}$, ma la forma: alla struttura "logica" si è infatti sostituita

\footnotetext{
${ }^{65}$ Simposio, 180 C $1-3$.

${ }^{66}$ Simposio, $185 \mathrm{E}-186 \mathrm{~B}$.

${ }^{67}$ Simposio, $188 \mathrm{E}$.

${ }^{68}$ Simposio, 193 D-E.

${ }^{69}$ Simposio, 193 E.
} 
quella "mitica". Soprattutto è forte il legame concettuale tra i due discorsi, in quanto il commediografo ha fornito l'elemento che mancava al discorso precedente (e così ha svolto il ruolo che gli aveva assegnato Erissimaco stesso di completarlo), in quanto il mito ruota intorno al fondamento unitario che è alla base della compresenza degli opposti. Solo che l'unità che Aristofane afferma è un principio assoluto, onniassorbente e distruttore delle differenze. Platone lo dimostra con un riferimento sconcertante:

E se ad essi, mentre giacciono insieme, si avvicinasse Efesto con i suoi attrezzi e domandasse loro: "Che cos'è, o uomini, che volete ottenere l'uno dall'altro?". E se essi non sapessero rispondere, e quegli domandasse ancora: "Forse è questo che volete: diventare la medesima cosa l'uno con l'altro, in modo che non vi dobbiate lasciare né giorno né notte? Se è questo che desiderate, io voglio fondervi e unirvi insieme nella medesima cosa, in modo che diventiate da due che siete uno solo, e finché vivrete, in quanto venite ad essere in questo modo uno solo, viviate insieme la vita, e quando morirete, anche laggiù nell'Ade, invece di due siate ancora uno, uniti insieme anche nella morte. Guardate se è questo che desiderate, e se vi basta ottenere questo". Sappiamo bene che, sentendo queste cose, neppure uno direbbe di no. Né direbbe di desiderare altro, ma direbbe di avere udito proprio quello che desiderava da tempo, ossia, congiungendosi e fondendosi insieme con l'amato, da due diventare uno $^{70}$.

Come si vede la situazione diviene paradossale perché questa unione comporta la totale scomparsa della singolarità anche per quanto riguarda l'anima, che sarebbe dissolta: quest'unità non salva le differenze, ma le distrugge tutte senza rispettare nessun livello ontologico. Una posizione che Platone non poteva in alcun modo avallare e che, non a caso, determina il rifiuto di Diotima.

${ }^{70}$ Simposio, 192 D 2 - E 9. 
Segue un nuovo intermezzo, con il richiamo ad Aristodemo che raccontava e uno scambio di battute, che isola il discorso di Agatone, certamente il più importante speculativamente in quanto chiede che si giudichi la cosa in sé e non gli effetti come hanno fatto gli altri oratori. Certo, il successivo svolgimento non è teoreticamente coerente con questa impostazione, ma Agatone non è un filosofo, ma un tragico. Tuttavia, le sue affermazioni sono di tale importanza che è da queste che Socrate riparte, discutendo proprio con il suo ospite.

Per affrontare adeguatamente il Simposio occorrono varie centinaia di pagine: spero solo che questo rapido riferimento abbia mostrato come la scrittura di Platone sia ricca a segnali che caratterizzano l'architettonica del dialogo.

$\mathrm{Ci}$ sia consentito riferire un altro evidente gioco, che riguarda Socrate. Lo svolgimento della "sua" trattazione di eros è diviso in tre parti: nella prima Socrate incalza e insegna ad Agatone; il dialogo prosegue con perfetta, totale conseguenzialita, con Diotima che incalza e insegna a Socrate; infine Diotima fa un lungo discorso e Socrate ascolta. Non mi interessa qui proporre una soluzione, ma di nuovo sottolineare i forti segnali che Platone ha dato al lettore e che qualsiasi interpretazione deve giustificare e valorizzare.

\section{Allusioni e inserimenti "estemporanei"}

Ci sono spesso, nel testo, passaggi il cui nesso con il resto appare del tutto misterioso. Eppure, se vengono adeguatamente approfonditi, questi inserimenti si rivelano significativi per la comprensione del dialogo.

Ne troviamo un esempio nello svolgimento del mito del Politico. Anche se meno famoso di tanti altri, si tratta di un grande racconto, che culmina in una grandiosa visione cosmologica basata sull'inversione del moto, che prefigura, miticamente, sia la protologia platonica, sia la sua visione cosmogonica, sia la funzione di pastore che spetta al solo Dio e che quindi va esclusa per il politico umano.

All'interno di questa trattazione compare all'improvviso una questione che non sembra collegarsi affatto al contesto e al dialogo nel suo complesso, il tema della felicità: 
Straniero - ...Ecco, Socrate, qual era la vita degli uomini sotto Crono. Di questa attuale, che la comune convinzione dice essere sotto Zeus, tu stesso ne hai esperienza in quanto sei qui presente. Potresti e vorresti giudicare quale delle due sia più felice?

SOCRATE IL GIOVANE - In nessun modo.

STRANIERO - Vuoi, allora, che in qualche maniera giudichi io per te?

SOCRATE IL GIOVANE - Certo.

STRANIERO - Se, dunque, gli allievi di Crono, avendo così tanto tempo e tanta facilità per poter dialogare non solo con gli uomini ma anche con le bestie, si servivano di tutto ciò per filosofare, discutendo sia con le bestie sia fra di loro, e domandando a tutte le creature se qualcuna, in possesso di una sua capacità specifica, conoscesse qualcosa di diverso dalle altre per arricchire il pensiero, è facile giudicare che gli uomini di allora superano di molto in felicità quelli di oggi. Se, invece, pieni a sazietà di cibo e di bevande, raccontavano l'uno all'altro ed alle bestie storie, come quelle che ancora oggi si raccontano di loro, anche questo è molto facile da giudicare, se devo esprimere francamente la mia opinione ${ }^{71}$.

L'affermazione, nella sua apparente inutilità, risulta chiara ed ha un primo effetto. Il dominio di Crono corrisponde a quella che solitamente viene chiamata l'età dell'oro, quella in cui tutti i beni sono garantiti all'uomo dalla natura e dalla attenzione provvidenziale degli dei. Ora Platone, rovesciando il giudizio tradizionale, sottolinea che la felicità non è garantita nemmeno da questo stato di benessere, perché tutto dipende dall'uso che del loro tempo hanno fatto quegli uomini. La conclusione, però, è che non è possibile rispondere perché ignoriamo che cosa davvero facessero.

L'inserimento resta quindi insoddisfacente e non trova in sé la spiegazione: Tuttavia, in questo modo il lettore ha ricevuto un

${ }^{71}$ Politico, 272 B 1 - D 2. 
segnale connesso al tema della felicità: non dovrebbero sfuggirgli gli altri due passaggi in cui il tema torna ad essere proposto, in modo altrettanto "incongruo". Ed è qui che Platone ci offre la giustificazione di questi inserimenti.

Nel corso della trattazione sulle costituzioni imitative, dopo averle classificate e averle considerate tutte solo imitazioni di quella unica e superiore vera costituzione scientifica che però risulta inapplicabile nella realtà umana, si osserva:

StRANIERO - Dunque, in quale di queste costituzioni non rette è meno difficile vivere, visto che è difficile in tutte, e in quale è più pesante? Bisogna che in qualche modo vediamo questo problema, anche se è un discorso accessorio rispetto al tema attuale. $\mathrm{Ma}$, in fondo, è forse per questo che noi tutti compiamo tutte le nostre azioni ${ }^{72}$.

Il punto decisivo è che Platone dice esplicitamente che il fine dell'azione umana è la felicità, che non è un tema rilevante e superiore come ad esempio il Bene, ma che è il vero motore delle nostre azioni. Pertanto, in un ragionamento sul comportamento umano come quello politico tale tema non può assolutamente essere dimenticato né in una esposizione mitica né nella riflessione teoretica.

Infatti, questa è la sottolineatura finale del dialogo stesso, nel momento in cui si celebra la possibile costituzione di uno stato il meno scorretto possibile, ad opera del vero politico:

StRANiERO - Diciamo, allora, che questo è il compimento del tessuto, ben intrecciato, dell'azione politica: la tecnica regia, prendendo il comportamento degli uomini valorosi e quello degli uomini equilibrati, li conduce a una vita comune, in concordia e in amicizia e, realizzando il più sontuoso e il migliore di tutti i tessuti, avvolge tutti gli altri, schiavi e

72 Politico, 302 B 7-9. 
liberi, che vivono negli stati, li tiene insieme in questo intreccio, e governa e dirige, senza trascurare assolutamente nulla di quanto occorre perché la città sia, per quanto possibile, felice ${ }^{73}$.

Il riferimento mitico non risulta allora inesplicabile, ma manifesta precocemente quello che è il tema, la domanda decisiva che sottende tutta la vita degli esseri umani.

\section{Il (cauto) utilizzo di altri dialoghi}

A volte è possibile trovare la spiegazione di alcune situazioni in altri dialoghi. Bisogna naturalmente muoversi con ancora maggiore cautela in queste occasioni, perché il rischio di inserimenti arbitrari è estremamente elevato. L'esempio che farò muove però da una situazione veramente paradossale.

Sul tema dell'insegnabilità della virtù, Platone polemizza duramente con politici e sofisti. Tale problema è affrontato senza esito, nel Protagora, in una serrata discussione tra Socrate e il sofista. Ora, com'è noto, il Menone si apre con lo stessa tema, imposto in modo quasi brutale, senza alcuna introduzione, con una domanda diretta. Il punto è che nel corso della discussione, non si ricorda affatto Protagora, ma Gorgia ${ }^{74}$ e il suo incontro con Socrate (cioè il dialogo che ha il titolo del sofista stesso): in quell'occasione forse si parlò della concezione gorgiana della virtù, ma Socrate non riesce a ricordare bene, per cui affida a Menone il compito di sostenere la parte del retore, visto che sicuramente ne condivide l'impostazione $^{75}$. Tuttavia, il giovane viene poi accusato di non voler ricordare la definizione gorgiana di virtù ${ }^{76}$.

La scena risulta quindi molto strana e difficilmente spiegabile: com'è possibile che entrambi non ricordino nemmeno per cenni che cosa Gorgia affermasse?

\footnotetext{
${ }^{73}$ Politico, 311 B 7 - C 6.

${ }^{74}$ Menone, $70 \mathrm{~B}-\mathrm{C}$.

${ }^{75}$ Menone, 70 C-D.

${ }^{76}$ Menone, 76 A-B.
} 
Visto che Platone stesso rimanda al Gorgia, la chiave può forse essere trovata in quel dialogo. Qui, contrariamente a quello che solitamente si sostiene, Gorgia viene trattato con molto rispetto ${ }^{77}$, anche sul piano personale. È infatti il sofista stesso che pone il problema etico ${ }^{78}$ che sottende l'utilizzo della forza propria del discorso: egli cerca di difendere il retore da accuse che dipendono dell'uso scorretto di queste tecnica. A conferma della sua personale rettitudine, Gorgia aggiunge che, se uno dei suoi allievi non sa nulla del giusto e dell'ingiusto, lo imparerà da lui ${ }^{79}$.

Quindi, Gorgia non afferma di insegnare esplicitamente etica, in quanto ne parla in termini pratici come un frutto naturale del suo stesso operare o comunque quasi come una funzione di supplenza. Infatti, nel dialogo omonimo, Menone dichiara che ha sempre apprezzato Gorgia perché si propone solo di rendere abili a parlare, mentre non promette di insegnare virtù e deride altri che lo fanno ${ }^{80}$.

Ma la questione è ben più grave per due ragioni: in primo luogo questa "apertura etica" costituisce il punto debole della posizione del sofista, quello che consente a Socrate di batterlo rapidamente e in modo oggettivamente umiliante, in quanto il maestro finisce con l'occupare una parte assai limitata del dialogo che pure porta il suo nome; nella parte restante intervengono il suo allievo Polo e il suo amico ed estimatore Callicle: ben triste ruolo per il più grande retore di quella generazione. Peggio ancora, i suoi allievi mostrano, in opere operato, il fallimento del suo insegnamento, perché via via si apre la strada un immoralismo che culmina nelle testi di Callicle.

In sintesi, Platone riconosce il soggettivo impegno di Gorgia, ma testimonia, sul piano teorico e su quello pratico, il suo fallimento.

${ }^{77}$ Per giustificare adeguatamente le affermazioni che mi appresto a fare, devo rimandare ad un mio studio: M. Migliori, Gorgia quale sofista di riferimento per Platone, «Giornale di metafisica», NS 21 (1999), pp. 101-126.

${ }^{78}$ Gorgia, 456 A - 457 C.

${ }^{79}$ Gorgia, 460 A.

${ }^{80}$ Menone, $95 \mathrm{C}$. 
Letta in questa chiave, l'apparente paradossalità del Menone acquista senso: i due interlocutori non ricordano che cosa Gorgia affermava della virtù perché il retore non ha una concezione etica degna di considerazione filosofica. La sua etica della situazione, puramente descrittiva, non è sufficiente ${ }^{81}$.

La situazione scenica, la dimenticanza, acquista così il senso di un giudizio: il sofista non possiede il concetto di virtù.

\section{L'utilità del metodo proposto}

Tutto il nostro discorso spinge a prestare un'attenzione al testo che è ben diverso da certo formalismo che affronta e isola la parola. Non è questione di filologia, ma di filosofia, platonica beninteso. Senza questo tipo di cura, si perdono segnali di notevole rilevanza e si finisce con l'attribuire a Platone posizioni che non sono le sue.

Un esempio di segnale famosissimo, soprattutto per la sua drammatica inutilità, è quello del Sofista. Mai parola fu più inutile di quella pronunciata dallo Straniero:

STRANIERO - Di questo, allora, ti prego ancora di più.

TEETETO - Di che cosa?

STRANIERO - Di non ritenere che io sia diventato una specie di parricida

TEETETO - Perché?

STRANIERO - Per difenderci, per noi sarà necessario sottoporre a prova il discorso del nostro padre Parmenide, e forzare il non-ente, sotto un certo rispetto, ad essere, e l'ente, a sua volta, sotto un certo rispetto, a non essere.

TEETETO - Evidentemente è questo il punto su cui dovremo dar battaglia nei nostri discorsi. ${ }^{82}$

\footnotetext{
${ }^{81}$ Per l'etica di Gorgia, cfr. quanto diciamo in M. Migliori, La filosofia di Gorgia. Contributi per una riscoperta del sofista di Lentini, CELUC, Milano 1973, pp. 118135.

82 Sofista, 2541 D 1-8.
} 
Qui abbiamo l'esplicita richiesta di non considerare l'operazione che sta iniziando come un parricidio, cioè come un totale rifiuto della posizione parmenidea. $\mathrm{Al}$ contrario si dice che per difendere quella posizione bisogna in qualche modo relativizzarla: per salvare l'essere e il vero dall'offensiva dei sofisti, bisogna ammettere che il non essere in qualche modo sia. Malgrado questa affermazione, non sono calcolabili i manuali e anche gli studi che parlano tranquillamente di parricidio, senza segnalare, magari solo con virgolette come abbiamo fatto sopra, la peculiare valenza del termine.

C'è un esempio meno noto, ma ancora più importante, almeno sul piano teoretico. La prima tesi del Parmenide riguarda l'UnoUno, per cui tutte le argomentazioni concludono con l'impossibilità di una qualsiasi predicazione. Il risultato è in qualche modo ovvio: l'Uno non partecipa dell'Essere, non è in alcun modo; di conseguenza non è nemmeno Uno: se infatti lo fosse, parteciperebbe dell'Essere. Pertanto non potrebbe avere qualcosa o essere qualcosa: non ha nome, né se ne ha definizione, né scienza alcuna.

Questa condizione, in realtà, potrebbe anche essere interpretata in senso positivo, come espressione della massima forma di trascendenza, sul modello che diverrà canonico nel neoplatonismo. Platone ha presente questa possibilità quanto meno perché conosce la trattazione eleatica, di cui questo Uno è manifestazione. Pertanto, proprio in questo caso, "costringe" il giovanissimo Aristotele a fare una affermazione perentoria, esprimendo l'unico giudizio che troviamo sull'esito delle 8 tesi.

"E' possibile dunque che questa sia la condizione dell'Uno?". "A me non pare possibile." 83

D'altra parte, questa negazione è ovvia, visto che la funzione dell'Uno dovrebbe essere quella di dare ragione del reale, mentre questo Uno non è in grado nemmeno di dare ragione di se stesso.

${ }^{83}$ Parmenide, 142 A 6-8. 
Non a caso Platone ci dà anche la ragione per cui un puro Uno non può essere a fondamento del reale:

Infatti una realtà nella sua interezza non potrà contemporaneamente avere la duplice funzione di subire e fare. Altrimenti l'Uno non sarebbe più Uno, ma due ${ }^{84}$.

Infatti, Platone abbandona immediatamente questa ipotesi dell'Uno-Uno e formalizza con una lunga trattazione una nuova ipotesi, quella dell'Uno-che-è ${ }^{85}$, che sarà utilizzata nell' "esercizio" svolto nelle tre successive tesi.

\section{BIBLIOGRAFIA}

\section{Platone}

(edizione critica e traduzione)

BURNET, J. Platonis Opera. Oxford, 1892-1906 (con varie edizioni).

REALE, G (ed.). Platone: Tutti gli Scritti. Milano: Bompiani, 2005.

\section{Bibliografia secondaria}

MIGLIORI, Maurizio. Arte politica e metretica assiologica: Commentario storico-filosofico al Politico di Platone. Milano: Vita e Pensiero, 1996.

. De la critique de Schleiermacher aux commentaires récents. Evolution et articulation du nouveau paradigme de Tubingen-Milan. “Études Philosophiques", 1998, p. 91-114.

. Dialettica e Verità: Commentario filosofico al Parmenide di Platone. Milano: Vita e Pensiero, 2000.

${ }^{84}$ Parmenide, 138 B 3-5.

${ }^{85}$ Parmenide, 142 B-C. 
. Gorgia quale sofista di riferimento per Platone. “Giornale di Metafisica", NS 21 (1999), p. 101-126.

. Il "Parmenide" e le dottrine non scritte di Platone. Istituto Suor Orsola Benincasa. Napoli, 1991.

. Il recupero della trascendenza platonica ed il nuovo paradigma. "Rivista di Filosofia Neoscolastica", 79 (1987), p. 351381.

. La filosofia di Gorgia. Contributi per una riscoperta del sofista di Lentini. Milano: Celuc, 1973.

. La scuola di Tubinga-Milano. "Il Cannocchiale", 1992, pp. 121-142.

. L'Idea del Bene nel Filebo. In: Studi in onore di Antonio Possenti. A cura di G. Almanza Ciotti, S. Balconcini, G.Mastrangelo Latini. Pisa-Roma: Istituti Editoriali Poligrafici Internazionali, 1998, p. 429-457.

Lo sviluppo tempestoso di un gioco compatto: la struttura del "Filebo". In: Il Filebo di Platone e la sua fortuna. A cura di P. Cosenza. Napoli: M.D’Auria Editore, 1996, p. 353-373.

L'uomo tra piacere, intelligenza e Bene. Commentario storico-filosofico al "Filebo" di Platone. Milano: Vita e Pensiero, 1998.

Sul Bene. Materiali per una lettura unitaria dei dialoghi e delle testimonianze indirette. In: New Images of Plato: Dialogues on the Idea of the Good. Edited by G. Reale and S. Scolnicov. Academia Verlag, Sankt Augustin, 2002, p. 115-149.

Tra polifonia e puzzle. Esempi di rilettura del "gioco" filosofico di Platone. In: La struttura del dialogo platonico: a cura di Giovanni Casertano. Napoli: Loffredo, 2000, p. 171-212. 
. Verso il filosofo: dialettica e ontologia nel "Sofista" di Platone. "Rivista di filosofia neo-scolastica", 91 (1999), p. 171-204.

Data de Registro 12/06/06

Data de Aceite 12/07/06 